\title{
Correction
}

\section{Correction: Wijetunge et al., Stimulated Emission Depletion (STED) Microscopy Reveals Nanoscale Defects in the Developmental Trajectory of Dendritic Spine Morphogenesis in a Mouse Model of Fragile X Syndrome}

In the article "Stimulated Emission Depletion (STED) Microscopy Reveals Nanoscale Defects in the Developmental Trajectory of Dendritic Spine Morphogenesis in a Mouse Model of Fragile X Syndrome” by Lasani S. Wijetunge, Julie Angibaud, Andreas Frick, Peter C. Kind, and U. Valentin Nägerl, which appeared on pages 6405-6412 of the April 30, 2014 issue, the authors regret that not all the funding sources were cited in the accepted version of the Acknowledgments. The corrected Acknowledgments are as follows: This work was supported by an international exchange grant from the Royal Society (P.C.K., U.V.N., L.S.W.), by proof-of-concept Eurobioimaging studies (L.S.W., U.V.N.), by the Medical Research Council (P.C.K.), by the Shirley Foundation (P.C.K., L.S.W.), by the Patrick Wild Centre (P.C.K., L.S.W.), by the institute National de la Santé et de la Médicale (A.F.), by the Agence Nationale pour la Recherche (Programme Chaires d'Excellence grant to U.V.N.), by Human Frontier Science Program (U.V.N.), by France-BioImaging (ANR-10INSB-04, U.V.N.), and by a NARSAD Young Investigator Grant from the Brain and Behavior Research Foundation (L.S.W.). The microscopy was performed at Bordeaux Imaging Center (BIC) of the Universite' de Bordeaux. We thank Philippe Legros (BIC) for technical support, and Jan Tonnesen (Université de Bordeaux), Tim O’Leary (Brandeis University), and Aleks Domanski (University of Edinburgh) for helpful discussions.

DOI: 10.1523/JNEUROSCI.2974-14.2014 